\title{
ON EVERTWHERE DENSE IMBEDDING OF FREE GROUPS IN LIE GROUPS
}

\author{
MASATAKE KURANISHT
}

In this note it will be proved that some kinds of Lie groups (including semisimple lie zroups) have an everywhere dense subgroup which is algebraically isomorphic to the free group generated by two elements (Theorem 8).

In $\$ 1$ characterizations of Lie groups which are approximated by discrete subgroups "are given. This section is closely connected with a part of the results of Malcev [1] and Matsushima [5], and some theorems are slight modifications of them (Theorerns 2 and 3 ).

In $\S 2$ a sufficient condition for Lie algebras to be generated by two elements is given, and in $\$ 3$ the main theorem is proved.

The writer owes very much to Messrs. Y. Matsushima, M. Gotô, and S. Mu. rakami for their discussions and suggestions during the preparation of this note.

§1. Lie groups approximated by discrete subgroups

THEOREM 1. Let $G$ be an n-dimensional local Lie group and $U$ a neighborhood of the identity $e$ of $G$, is which a canonical coordinates sysien is introduced. Let $H$ be a discreie subsei of $G$ satisfying the following conditions,

1) If $V$ is any neighborhood of $e$ such that $V V^{-1} \subset U$, then for any $x$, $y \in V \cap H, x y^{-1} \in H$.

2) $I l$ conlains $h_{1}, h_{2}, \ldots, h_{n}$, which are linearly independeni in $U$ (with respect to the coordinates system in $U$ ).

Then $G$ is a nilpotent lie gronp.

Proof, It is easily seen that if $x$ and $y$ are elements of $U$, then

$$
\left|x y x^{-1} y^{-1}\right| \lesseqgtr \min (|x|,|y|) \text {, }
$$

where $|x|$ is the euclidean distance between $e$ and $x$ in $U$. Let $y$ be the point of $H$ which is not equal to $e$ and $|p| \leqq|x|$ for every $x \in H$. Then by (1) $p$ is commutative with every element of $H$, in particular

Hence

$$
h_{i} p h_{i}^{-1}=p \quad(i=1,2, \ldots, n) \text { 。 }
$$

$$
h_{i} p^{\lambda} h_{i}{ }^{-1}=p^{\lambda ?)} \quad(i=1,2, \ldots, n),
$$

Received Oct, 25, 1950。

1) Cf. Definition 1. This notion is introduced by H. Tôyama [7].

2) Let $x^{\lambda}$ be the one-parameter subgroup passing $x$ such that $x^{1}=x$. We use this notation throughout this note. 


$$
\therefore \quad p^{-\lambda} h_{i} p^{\lambda}=h_{i} \quad(i=1,2, \ldots, n) .
$$

By the same argument as above, we can deduce

$$
p^{-\lambda} h_{i}{ }^{\mu} p^{\lambda}=h_{i}{ }^{\mu} \quad(i=1,2, \ldots, n) .
$$

Hence $p$ is contained in the center of $G$. Since $H$ is a finite set, it is easy to see that $G / p^{\lambda}$ is an $(n-1)$-dimensional local Lie group satisfying the conditions of our Theorem. By the assumption of the induction, $G / p^{\lambda}$ is a nilpotent Lie group and $p^{\lambda}$ is contained in the center of $G$. Hence $G$ is also nilpotent.

DefinITION 1. A locally compact group $G$ is said to be approximated by discrete subgroups if there is a sequence of discrete subgroups $D_{n}(n=1,2, \ldots$, , of $G$ satisfying the following condition $(A)$.

$(A)$ For any open set $O$ of $G$, there exists an integer $I$ (depending on $O$ ) such. that $O \cap D_{n}$ is not empty for every $n \geqq 1$.

COROLlaRy. Let $G$ be a Lie group approximated by discrete subgroups. Then $G$ is nilpotent.

Proof is immediate from Theorem 1.

DEFINITION 2. Lie algebras are said to be rational if the structure constants are all rational numbers under the appropriated basis. Lie groups are said to be rational if Lie algebras corresponding to $G$ are rational.

The following two Lemmas are well known.

Lemma 1. Let $G$ be a rational, nilpotent, and simply connected Lie group. ${ }^{3)}$ Then the space of $G$ is homeomorphic to a Euclidean space, i.e., we can introduce in $G$ a cartesian coordinates system $\left(x^{1}, \ldots, x^{n}\right)$ satisfying the following conditions :

1) The multiplication functions, $f^{i}\left(x^{1}, \ldots, x^{n} ; y^{1}, \ldots, y^{n}\right)(i=1,2, \ldots, n)$, expressed in these coordinates are polynomials of $x^{1}, \ldots, x^{n} ; y^{1}, \ldots, y^{n}$ with rational coefficients.

2) $g(t)=\left(t x^{1}, \ldots, t x^{n}\right)$ with fixed $\left(x^{1}, \ldots, x^{n}\right)$ is a one parameter subgroup of $G$.

Lemma 2. Let $x^{1}, \ldots, x^{n}$ be a coordinates system of $G$ satisfying the conditions of Lemma 1. If $x$ is an element of the center of $G$, then

$$
x \cdot y=x+y
$$

for every element $y$ of $G$.

DEFINITION 4. The coordinates system satisfying the conditions of Lemma 1

3) It is known that there exist nilpotent $\mathrm{Lie}$ algebras which are not rational. $\mathrm{Cf}$. Malcev [4]. 
is said to be rational. An element $x=\left(x^{1}, \ldots, x^{n}\right)$ is said to be rational (in the rational coordinates system) if $x^{i}(i=1, \ldots, n)$ are rational numbers. $A$ subgroup $D$ is said to be rational (in the rational coordinates system) if all the elements of $D$ are rational elements.

THEOREM 2. Lei $G$ be an n-dimensional, nilpoient, simply connected, and rational Lie group. Introduce a rational coordinates system in $G$. Suppose that there is given a rational discrete subgroup $D$. Then $G$ is approximated by discrite suhgroups $D_{m}$ containing $D$. Moreover, for every $D_{m}$, there exist $d_{1}^{(m)}, \ldots, d_{n}{ }^{(m)}$ in $D_{m}$ such that every element $d$ of $D_{m}$ can be written uniquely as

$$
d=\breve{d}_{1}^{(m)^{a_{1}}}, \ldots, d_{n}^{(m)^{\left(b_{i}\right)}} \text {. }
$$

Proof. (By induction on the dimension of $G$ ). Since $D$ is nilpotent, there exists an element $d_{c}$ of $D$, which is commutative with every elements of $D$. Since the one-parameter subgroup passing an element is unique in $G, d_{c}$ is con tained in the center of $G$. We can assume without loss of generality that $d_{c}=(1,0, \ldots, 0)$ and $x=(\xi, 0, \ldots, 0),(0<|\xi|<1)$ is not contained in $D$. Cet $Z$ be the one-parameter subgroup passing $d_{c} . G / Z$ is also nilpotent, rational, and simply connected Lie group, and by associating to the coset containing $x=\left(x^{1}, \ldots, x^{n}\right)$ the point $\left(x^{2}, \ldots, x^{n}\right)$ of $(n-1)$-dimensional euclidean space, a rational coordinates system is introduced in $G / Z$." Let $I^{\prime}$ be the image of $D$ in $G / Z$. Then $G / Z$ satisfies the condition of our Theorem replacing $D$ by $D^{\prime}$. By the assumption of induction $G / Z$ is approximated by $D_{n}^{\prime}$ contairing $D^{\prime}$. Let $d_{1}^{\prime}, \ldots, d_{n-1}^{\prime}{ }^{5}$ be the generaters of $D_{m}^{\prime}$ as stated in the 'Theorem, and put $d_{i}=\left(0, d_{i}{ }^{\prime 2}, \ldots, d_{i}{ }^{\prime n}\right)\left(d_{i}{ }^{\prime}=\left(d_{i}{ }^{\prime 2}, \ldots, d_{i}{ }^{\prime n}\right), i=1,2, \ldots, y, \ldots 1\right)$

Then

$$
d=\prod_{i=1}^{n-1} d_{i}^{m_{i}} \cdot \prod_{i=1}^{n-1} d_{i}^{h_{i}}=g \cdot \varphi\left(m_{1}, \ldots, m_{n-1} ; h_{1}, \ldots, h_{n-1}\right),
$$

where $\varphi\left(m_{1}, \ldots, m_{n-1} ; h_{1}, \ldots, h_{n-1}\right) \in Z, g=\left(0, g^{n}, \ldots, g^{n}\right)$, and $\left(g^{2}, \ldots, g^{n}\right)$ $\in D_{m}{ }^{\prime}$. Since by Lemma $2 \varphi$ is the first coordinate of $d, d_{i}{ }^{m_{i}}=\left(0, m_{i} d_{i}{ }^{2}, \ldots\right.$, $\left.m_{i} d_{i}{ }^{n}\right)$, and the product functions are polynomials with rational coefficients, it is verified that there exists $d_{n}=(1 / M, 0, \ldots, 0)$ ( $M$ is an integer) such that $\varphi$ $\left(m_{1}, \ldots, h_{n-1}\right)=d_{n} a_{m_{1}}, \ldots, h_{n-1}$ with $a_{m_{1}}, \ldots, h_{h-1}$ as an integer. If we take $M$ sufficiently large, the group $D_{m}$ generated by $d_{1}, \ldots, d_{n}$ contains $D$, and $\left\{D_{m}\right\}$ approximates $G$.

TheOREM 3.6) Let $G$ be a nilpotent and simply connected Lie group and $D$ a discrete subgroup of $G$. Assume that there is no connected proper subgroup

4) Cf. Lemma 2.

5) We omit the index $(m)$ to avoid the inessential complications.

6) For the proof see Malcev [4], or Theorem of Matsushima [5]. 
$H$ of $G$ containing $D$. Then $G$ is rational and under the appropriated rational coordinates system $D$ is a rational group.

THEOREM 4. Let $G=\widetilde{G} / Z$ be a connected Lie group, where $\widetilde{G}$ is the universal covering group of $G$, and $Z$ is a discrete normal subgroup of $\widetilde{G}$. The necessary and sufficient conditions for $G$ to be approximated by discrete subgroups are the following conditions.

1) $G$ is nilpotent and rational Lie group.

2) Under the appropriated rational coordinates sysiem, $Z$ is a rational subgroup.

Proof is immediated from the corollary to. Theorem 1 and from Theorem 2.

THEOREM 5. Let $G$ be a connected Lie group. The necessary and sufficient condition for $G$ to be approximated by discrete subgroups are that there exists a discrete subgroup $D$ of $G$ satisfying the following condition.

There exists a neighborhood $U$ of e which can be identified with neighborhood of the corresponding Lie algebra $L$ and $U \cap D$ contains the basis of $L$.

Proof. Suppose that there exist $D$ and $U$ satisfying the above condition. By Theorem $1, G$ is nilpotent. Let $\widetilde{G}$ be the universal covering group of $G$ and $\widetilde{D}$ be the subgroup of $\widetilde{G}$ covering $D$. Then by Theorem $3, \widetilde{G}$ and $\widetilde{D}$ are rational. Hence by Theorem $2, \widetilde{G}$ is approximated by. $\widetilde{D}_{n}$ containg $\widetilde{D}$. Since $\widetilde{D}$ contains $Z,{ }^{\prime \prime} G$ is approximated by discrete subgroups $D_{n}$ which are the images of $\widetilde{D}_{n}$.

Remark. There exist rational nilpotent Lie groups which cannot be approximated by discrete subgroups. For example we can easily construct such a Lie group whose center is one-dimensional.

\section{\$2. Lie algebras generated by two elements}

Let $L$ be a Lie algebras over a field $F$ of characteristic $0 . L$ is said to be generated by two elements $a$ and $b$ if the minimal Lie subalgebra containing $\lambda a$ and $\mu b(\lambda, \mu \in F)$ is $L$, Let $\bar{F}$ be the algebraic closure of $F$, and let $\bar{L}$ be the Lie algebra obtained from $L$ by extending $F$ to $\vec{F}$. If $L$ is semi-simple, so is $\bar{L}$, and vice versa. It is well known that the semi-simple lie algebra $L$ has the following structure. Let $\bar{H}$ be a maximal abelian subalgebra of $\bar{L}$ containing a regular element and let $h_{1}, \ldots, h_{l}(\in L)$ be a basis of $\bar{H}$. Then there exists a system of vectors of an $l$-dimensional euclidean space, whose vectors are called root vectors. To each root vector $\alpha$ we can corelate an element $e_{\alpha}$ of

7) Suppose that $\widetilde{G}=G / Z$. 
$I$ so that $h_{1}, \ldots, h_{l}, e_{\alpha}, e_{\beta}, \ldots$ constitute a basis of $\bar{L}$, and the structure formulaes of $\bar{L}$ have the following form

$$
\left[h, e_{\alpha}\right]=(\alpha \lambda) e_{\alpha},
$$

where $h=\sum_{i=1}^{l} \lambda^{i} h_{i}, \alpha=\left(\alpha_{1}, \ldots, \alpha_{l}\right),(\alpha \lambda)=\sum_{i=1}^{l} \alpha_{i} \lambda^{i}$.

$$
\begin{aligned}
& {\left[e_{\alpha}, e_{\beta}\right]= \begin{cases}0 & \alpha+\beta \neq 0 \text { is not a root, } \\
N_{\alpha, \beta} e_{\alpha+\beta}, N_{\alpha, \beta} \neq 0, & \alpha+\beta \neq 0 \text { is a root, }\end{cases} } \\
& {\left[e_{\alpha,} e_{-\alpha}\right]=h_{\alpha}=\sum_{i=1}^{b} \alpha^{i} h_{i} .}
\end{aligned}
$$

Root vectors are distinct among each other, and if $\alpha$ is a root vector, then $-\alpha$ is also a root vector. There exist root vectors $\alpha^{(1)}, \ldots, \alpha^{(l)}$ such that $\left[e_{\alpha^{(1)}}, e_{-\alpha^{(1)}}\right]$, . . , $\left[e_{a^{(l)}}, e_{-a^{(l)}}\right]$ form a basis of $\bar{H}$.

THEOREM 6. Let $L$ be a semi-simple Lie algebra over a field $F$ of characteristic 0 . Then there exist two elements $a$ and $b$ which generate $L$.

Proof. Pui $a=\sum_{\alpha} e_{\alpha}$ and $h=\sum \lambda^{i} h_{i}$. Then

$$
\begin{aligned}
& s_{1}=[h, a]=\sum_{\alpha}(\alpha \lambda) e_{\alpha}, \\
& s_{2}=[h,[h, a]]=\sum_{\alpha}(\alpha \lambda)^{2} e_{\alpha,} \\
& s_{k}=[h, \ldots,[h, a] \ldots]=\sum(\alpha \lambda)^{k} e_{\alpha} .
\end{aligned}
$$

Take $\lambda=\left(\lambda^{1}, \ldots, \lambda^{l}\right)$ such that $((\alpha-\beta) \lambda) \neq 0$ for every root $\alpha$ and $\beta$. Then $a, s_{1}, s_{2}, \ldots, s_{n-l-1}$ are linearly independent. Let $L^{*}$ be the minimal subalgebra over $\bar{F}$ of $\bar{L}$ containing them. Then $L^{*}$ contains each $e_{\alpha}$, hence it contains also $\left[e_{a^{(i)}}, e_{-\alpha^{(i)}}\right], i=1,2, \ldots, l_{\text {. }}$ Thus $L^{*}=L$.

To summarize the above results, there exist finite number of monomials $P_{i}(x, y)=[\ldots[x y] \ldots]$ such that every element of $L$ is a linear combination of $P_{i}(a, h)$ with coefficients of $\bar{F}$. Take a basis $p_{1}, \ldots, p_{n}$ of $L$ and put

$$
\sum_{k=1}^{n} r_{i}^{k} P_{k}=P_{i}\left(\sum_{j=1}^{n} \xi^{j} p_{j}, \sum_{j=1}^{n} \eta^{j} p_{j}\right) \text {. }
$$

Then $r_{i}^{k}$ are polynomials of $\xi^{i}, r_{i}^{j}$ with coefficients in $F$. Let $M\left(\xi^{1}, \ldots, \xi^{n} ; \eta^{1}\right.$; $\left.\ldots, \eta^{n}\right)$ be the matrix $\left(\gamma_{i}{ }^{k}\right)$ and $a=\sum_{i=1}^{n} a^{i} p_{i}, h=\sum_{i=1}^{n} b^{i} p_{i}$. Then the rank of $M\left(a_{1}, \ldots, a^{n} ; b^{1}, \ldots, b^{n}\right)$ is $n$. Hence there exist elements $c^{1}, \ldots, c^{n}, d^{1}$, $\ldots, d^{n}$ of $F$ such that the rank of $M\left(c^{1}, \ldots, c^{n} ; d^{1}, \ldots, d^{n}\right)$ is also $n$. Then $L$ is generated by $c=\sum_{i=1}^{n} c^{i} p_{i}, d=\sum_{i=1}^{n} d^{i} p_{i}$.

Remark 1. Let $L$ be a Lie algebra over a field $F$ of characteristic 0 。 By Levi's theorem $L$ is decomposed into the form

$$
L=S+R, \quad S \cap R=0,
$$

where $R$ is the radical of $L$ and $S$ is a semi-simple subalgebra. Associating a linear transformation: $R \ni x \rightarrow[s, x] \in R$ for every element $s$ of $S$, a representa- 
tion of $S$ is given. The representation of $S$ is characterized by its weights. If the weights of this representation satisfy some conditions, the proof of Theorem 6 is also applicable for these Lie algebras.

\section{§3. Groups generated by two elements}

A topological group is said to be generated by two elements $a$ and $b$, if the minimal closed subgroup containing $a$ and $b$ is $G$.

THEOREM 7. Let $G$ be a connected and perfect Lie group and $L$ the Lie algebra corresponding to $G$. If $L$ is generated by two elements, so is $G$. Moreover, we can take the generators $a$ and $b$ in an arbitrary small neighborhood of the identity $e$.

Proof. Since $L$ is generated by two elements, there exist $a$ and $b$ in an arbitrary small neighborhood of $e$, such that $a^{\lambda}$ and $b^{\lambda}$ generate $G$. Denote by $H_{n}$ the closed subgroup generated by $a^{1 / 2^{n}}$ and $b^{1 / 2^{n}}$, and let $H_{n}{ }^{0}$ be the component of the identity in $H_{n}$, then

$$
\begin{aligned}
& H_{1} \subset H_{2} \subset \ldots, \quad H_{1}{ }^{0} \subset H_{2}{ }^{0} \subset \ldots, \\
& \bigcup_{n=1}^{\infty} H_{n}=G .
\end{aligned}
$$

Since $H_{n}{ }^{0}$ is connected, there exists an integer $N$ such that $H=H_{N}{ }^{0}=H_{N+1}^{0}=\ldots$, and so for every element $h$ of $H_{n}, n=1,2, \ldots$

$$
h^{-1} H h=H \text {. }
$$

From (1) and (2), $H$ is a closed normal subgroup of $G$ and $G / H$ is approximated by discrete subgroups $H_{N+k} / H, k=0,1,2, \ldots$ By Theorem $1 G / H$ is nilpotent, but on the other hand $G$ is perfect. Hence $G / H=\{e\}$. This shows that $G$ is generated by $a^{1 / 2^{-V}}$ and $b^{1 / 2^{N}}$.

Our next aim is to take generators $a$ and $b$ which do not satisfy any relations. For that purpose we make some preparations.

Let $A$ be a compact metric space and $\mathfrak{F}(A)$ the family of all the closed subsets of $A$. Let $U_{\varepsilon}(B)$ be the $\varepsilon$-neighborhood of $B \subset A$. For $M$ and $N$ $\in \mathfrak{F}(A)$, put

$$
\alpha(M, N)=\inf _{\substack{C \varepsilon(M) \geq N \\ U \varepsilon(N) \geq}} \varepsilon .
$$

It is well known ${ }^{s)}$ that by this (Hausdorff) metric $\alpha, \mathfrak{F}(A)$ becomes a compact space.

Lemma 3. Let $G$ be a connected perfect Lie group. If a and $b$ generate $G$, then there exists a neighborhood $V$ of $e$ such that any $a^{\prime} \in V a$ and $b^{\prime} \in V b$ generate $G$.

8) Cf. Chapter 2, §5 of Alexandroff and Hopf [1]. 
Proof. Suppose that there exists no neighborhood $V$ of $e$ as stated in the Lemma. Then there exist two sequences $\left\{a_{n}\right\}$ and $\left\{b_{n}\right\}$ converging to $a$ and $b$ respectively, such that the closed subgroups $G_{n}$ generated by $a_{n}$ and $b_{n}$ are proper subgroups of $G$.

Take a neighborhood $U$ of $e$ in which we can introduce a canonical coordinates system, and put

$$
K_{n}=G_{n} \cap \bar{U}
$$

Since $K_{n} \in \mathscr{F}(\bar{U})$, we can select a subsequence $\left\{K_{n^{\prime}}\right\}$ of $\left\{K_{n}\right\}$ converging to $K$ $E \tilde{F}(\bar{U})$ by the above metric $\alpha$. Since $a$ and $b$ generate $G$, it is not hard to show that $K=\bar{U}$. Hence there exists an integer $N$ such that $K_{N}$ contains $r^{9)}$ linearly independent points $p_{i}(i=1,2, \ldots, r)$ of $K=\bar{U}$. Let $G_{N^{0}}{ }^{\text {be the com- }}$ ponent of the identity in $G_{N}$. Then

$$
p_{i}^{-1} G_{N}{ }^{0} p_{i} \subset G_{N}{ }^{0} \quad \text { for } i=1,2, \ldots, r .
$$

Hence $G_{N}{ }^{0}$ is the normal subgroup of $G$. In the factor group $G / G_{N}{ }^{0}$ the discrete subgroup $G_{N} / G_{N}{ }^{0}$ satisfies the conditions stated in Theorem 5 , hence $G / G_{x^{n}}$ is a nilpotent Lie group. This is a contradiction to the fact that $G$ is a perfect group.

Lemma 4. Let $G$ be a Lie group which is not solvable and let $U$ be a neighborhood of $e$ in which we can introduce the canonical coordinates system. Suppose that there exist open sets $O$ and $O^{\prime}$ contained in $U$, a monomial $M(x, y)$

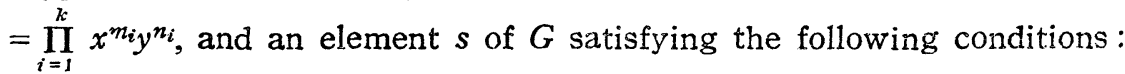

For every $p \in O$ and $q \in O^{\prime}, M(p, q)=s$. Then the monomial $M(x, y)$ is trivial.

Proof. I) Suppose at first that $G$ is a semi-simple linear group. Let $L$ be the Lie algebra of matrices corresponding to the linear group $G$. Then $\bar{L}=\left\{\lambda x^{*} \mid x^{*}\right.$ $\in L, \lambda$ : complex number $\}$ is a complex semi-simple Lie algebra. Every element $x \in U$ can be written as $\left(x_{j}{ }^{i}\right)^{\lambda}=x^{\lambda}=\exp \lambda x^{*}, x^{*}=\left(x^{*}{ }_{j}{ }^{i}\right) \in L . \quad\left\{x \mid x=\exp x^{*}\right.$, $\left.x^{*} \in \bar{L}\right\}$ generates a Lie group $\widetilde{G}$ containing $G$. Fix $p=\exp p^{*} \in O, q \in O^{\prime}$, and $x=\exp x^{*}$ of $G$, and define

$$
x_{t}=\exp \left(t p^{*}+(1-t) x^{*}\right), t: \text { complex number, }
$$

and

$$
\left(f_{j}^{i}(t)\right)=M\left(x_{t}, q\right) \text {. }
$$

Then $f_{j}^{i}(t)$ is an analytic function of $t$ and is constant $\left(=s_{j}{ }^{i}\right)$ if $t$ is real and sufficiently near to 1 . Hence $f_{j}^{i}(t)$ is constant for every value of $t$, that is, $M(x, q)=s$ for every element $x=\exp x^{*}, x^{*} \in L$. Define

$$
x^{\lambda}=\exp \lambda x^{*}, x^{*} \in L
$$

9; Let $r$ be the dimension of $G$. 
and

$$
\left(g_{j}^{i}(\lambda)\right)=M\left(x^{\lambda}, q\right) .
$$

Then $g_{j}{ }^{i}(\lambda)$ is an analytic function of the complex number $\lambda$ and constant for real value of $\lambda$, hence $M(x, q)=s$ for $x=\exp x^{*}, x^{*} \in \bar{L}$. Repeating these arguments, we can deduce that

$$
M(x, y)=s \text { for any elements } x=\exp x^{*}, y=\exp y^{*}, x^{*} \in \bar{L}, y^{*} \in \bar{L} .
$$

But every complex semi-simple Lie algebra contains a subalgebra which is isomorphic to the unimodular Lie algebra of degree 2. Hence it is sufficient to prove the case when $G$ is the unimodular group of degree 2. Put ${ }^{10}$

$$
x=\left(\begin{array}{ll}
1 & 1 \\
0 & 1
\end{array}\right), \quad y^{\lambda}=\left(\begin{array}{ll}
1 & 0 \\
\lambda & 1
\end{array}\right),
$$

then

$$
\begin{gathered}
M\left(x, y^{\lambda}\right)=\prod_{i=1}^{k}\left(\begin{array}{rr}
1 & m_{i} \\
0 & 1
\end{array}\right)\left(\begin{array}{ll}
1 & 0 \\
n_{i} \lambda & 1
\end{array}\right)=\left(\begin{array}{ll}
g(\lambda) & * \\
* & *
\end{array}\right), \\
g(\lambda)=m_{1} n_{1} \ldots m_{k} n_{k} \lambda^{k}+\ldots
\end{gathered}
$$

Hence if $M(x, y)$ is not trivial, $F(x, y) \neq s$ for some $x=\exp x^{*}, y=\exp y^{*}$ of $G$.

(II) Suppose that $G$ is a semi-simple Lie group. Then there exists a discrete normal subgroup $Z$ such that $G / Z$ is isomorphic to a linear group. Consulting (I) and the discreteness of $Z$, it is easy to prove the Lemma in this case.

(III) Let $G$ be a Lie group which is not solvable. Let $R$ be the radical of $G$. Then $G / R$ is semi-simple and $\neq\{e\}$. Suppose that the monomial $M(x, y)$ satisfies the conditions of our Lemma for $G$, then $M(x, y)$ also satisfies the same conditions for $G / R$. Hence by (II) $M(x, y)$ is trivial.

THEOREM 8. Let $G$ be a connected perfect Lie group. Suppose that the Lie algebra corresponding to $G$ is generated by two elements. "1) Then $G$ coniains an everywhere dense free group with two generaters, that is, there exists a subgroup $F$ of $G$ satisfying the following conditions.

(1) $F$ is everywhere dense in $G$.

(2) $F$ is isomorphic to a free group $\mathfrak{F}$ generated by two elements.

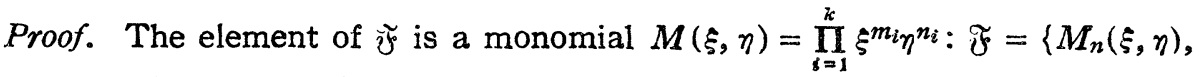
$n=1,2, \ldots\}$ Let $A_{n}=\left\{(x, y) \mid M_{n}(x, y)=\Pi x^{m_{i}} y^{n_{i}}=e,(x, y) \in G \times G\right\}$,

By Theorem $6, G$ is generated by two elements $p$ and $q$. By Lemma 3, there exist open sets $P \exists p$ and $Q \ni q$ such that $p^{\prime}$ and $q^{\prime}\left(p^{\prime} \in \bar{P}, q^{\prime} \in \bar{Q}\right)$

10) See Hayashida [2].

11) Consulting the remark 1 of Theorem 5, we can construct perfect Lie algebras which can not be generated by two elements. 
generate $G$. By Lemma $4, A_{n} \cap(\bar{P} \times \bar{Q})$ is nowhere-dense in $\bar{P} \times \bar{Q}$. Hence by Baire's theorem, there exists $(\bar{p}, \bar{q}) \in \bar{P} \times \bar{Q}$ such that $(\bar{p}, \bar{q}) \notin \bigcup_{n=1}^{\infty} A_{n}$. Let $F$ be the subgroup of $G$ algebraically generated by $\bar{p}$ and $\bar{q}$, then $F$ satifies our conditions.

COROLLARY. Every connected semi-simple Lie group contains an everywhere dense free group with two generators.

\section{REFERENCES}

[1] Alexandroff and Hopf, Topologie, Springer, (1935).

[2] Hayashida, On faithful representations of free groups, Kôdai Math. Sem. Rep. No. 2, p. 27, (1949).

[3] Kuranishi, Two elements generations on semi-simple Lie groups, Kôdai Math. Sem. Rep. Nos. 5 and 6, pp. 9-10 (1949).

[4] Malcev, On a class of homogeneous space, Izvestiya Akad. Nauk SSR. Ser. Math. 13 (1949) (in Russian).

[5] Matsushima, On the discrete subgroups and homogeneous spaces of nilpotent Lie groups (in this issue).

[6] Pontrjagin, Topological groups, Princeton, (1939).

[7] Tôyama, On discrete subgroups of a Lie group. Kòdai Math. Sem. Rep. No. 2, pp. 36-37, (1949).

Mathematical Institute, Nagoya University 